\title{
On the Intrinsic Optical Absorptions of Tetrathiafulvalene Radical Cations and Isomers
}

\author{
Maj-Britt Suhr Kirketerp, ${ }^{\dagger}$ Kristine Kilså, ${ }^{\ddagger}$ Leonardo Andrés Espinosa Leal, ${ }^{\S}$ Daniele Varsano,, $\mid l$ Angel \\ Rubio, ${ }^{\star \S}$ Mogens Brøndsted Nielsen ${ }^{\star, \neq}$, Steen Brøndsted Nielsen ${ }^{\star, \dagger}$ \\ ${ }^{\dagger}$ Department of Physics and Astronomy, Aarhus University, Ny Munkegade, DK-8000, Århus C, Denmark \\ ${ }^{\S}$ Nano-bio Spectroscopy Group , Department of Material Science, University of the Basque Country and Materials \\ Physics Center (CSIC-UPV/EHU), Av. Tolosa 72, E-20018 Donostia-San Sebastián, Spain \\ "Department of Physics, University of Rome 'La Sapienza', Italy \\ ${ }^{*}$ Department of Chemistry, University of Copenhagen, Universitetsparken 5, DK-2100 Copenhagen Ø, Denmark
}

RECEIVED DATE (automatically inserted by publisher); angel.rubio@ehu.es, mbn@kiku.dk, sbn@phys.au.dk

\begin{abstract}
The intrinsic optical properties of tetrathiafulvalene, tetrathianaphthalene and tetramethylthiotetrathiafulvalene radical cations were elucidated by state-of-the-art gas phase action spectroscopy, theoretical calculations and spectroelectrochemistry. We show unambiguously that the low-energy absorptions of tetramethylthiotetrathiafulvalene and tetrathianaphthalene cations in solution are an intrinsic absorptions and do not result from a $\pi$-dimers.
\end{abstract}

Tetrafulvalene (TTF, Chart 1) is a redox-active molecule that has been explored considerably in supramolecular chemistry, molecular electronics, and materials science. ${ }^{1}$ The ability of tetrathiafulvalene and alkylthio-substituted TTF radical cations to form $\pi$-dimers $\left(\mathrm{TTF}_{2}{ }^{2+}\right)$ has been subject of some controversy. The parent TTF radical exhibits an absorption maximum at a longestwavelength absorption of $580 \mathrm{~nm}$ in acetonitrile, ${ }^{2}$ which has been assigned to an intrinsic absorption of the cation. However, in ethanol at $225 \mathrm{~K}$, a new absorption at $714 \mathrm{~nm}$ can be observed, and it was interpreted as formation of $\pi$-dimers. ${ }^{3}$ This assignment was confirmed by Khodorkovsky and co-workers ${ }^{4}$ by ESR studies and calculations. It was recently shown by Sallé and co-workers ${ }^{5}$ that dimerization can be enforced between two closely situated TTFs in a calixarene assembly, and along the same line Stoddart and co-workers have observed it in a bistable tripodal[4] rotaxane. ${ }^{6}$ Thus, $\pi$-dimerization is an intriguing design element for supramolecular chemistry, but the substitution pattern of the TTF does in fact seem to play a major role for its ability to dimerize. For the radical cation of tetramethylthiotetrathiafulvalene (TMTTTF, Chart 1) and related derivatives, an absorption around 843 $\mathrm{nm}$ in $\mathrm{MeCN}$ at room temperature was in several studies ${ }^{7}$ interpreted as a $\pi$-dimer absorption. Studies on a bis-TTF macrocycle showed the absorption in this case to be concentration dependent (in comparison to a higher energy absorption), which was taken as evidence of the intermolecular character of the transition. ${ }^{7 c}$ Nevertheless, ESR and calculation studies by Khodorkovsky et al. ${ }^{4}$ convincingly showed that TMT-TTF radical cations do not dimerize.

We became interested to shed further light on the intrinsic absorption properties of TTF radical cation by studies in the gas phase, as such studies would both provide the isolated molecule characteristics and by comparison with solution phase experiments reveal any possible solvent influence on the absorption maxima. In addition to $\mathrm{TTF}^{\bullet+}$ and $\mathrm{TMT}^{-\mathrm{TTF}^{\bullet+}}$, we have studied the properties of tetrathianaphthalene (TTN, Chart 1 ), which is an isomer of the parent TTF. The measured gas phase and solution phase properties of these cations were compared to calculated ones. In the calculational study, a third possible isomer of TTF was included, a fused dithiafulvene (FDTF, Chart 1).

Chart 1. Sulfur-heterocvcles

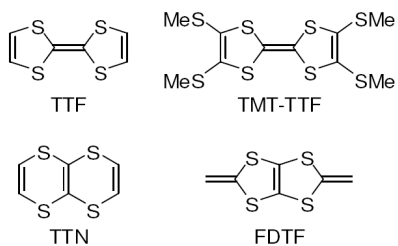

It has previously been shown ${ }^{\gamma}$ that TTN undergoes one reversible oxidation and a subsequent irreversible oxidation, but to our knowledge the absorption properties of the radical cation were never measured. For this reason we performed spectroelectrochemical studies on TTN. The cyclic voltammogram is shown in the inset of Figure 1. A reversible oxidation was found at $E_{0}\left(\mathrm{TTN}^{+} / \mathrm{TTN}\right)=0.36 \mathrm{~V}$ (half-wave potential) vs. $\mathrm{Fc}^{+} / \mathrm{Fc}$ and an irreversible oxidation at $E_{\mathrm{p}}\left(\mathrm{TTN}^{2+} / \mathrm{TTN}^{+}\right)=1.01 \mathrm{~V}$ vs. $\mathrm{Fc}^{+} / \mathrm{Fc}$. Spectroelectrochemistry revealed an absorption maximum of $\mathrm{TTN}^{\bullet+}$ at ca. $900 \mathrm{~nm}$ (Figure 1). We note that bulk electrolysis was not completely reversible as upon returning to the neutral species some other absorption features emerge, in addition to the expected disappearance of the radical cation absorption band.

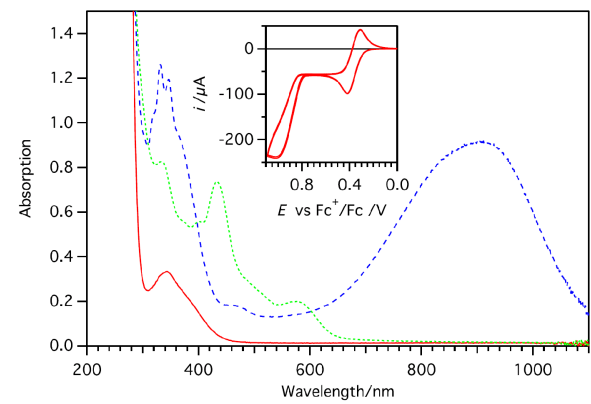


Figure 1. Absorption spectra of the neutral TTN (red solid line) and the oxidized species, $\mathrm{TTN}^{\bullet+}$ (blue dashed line). Spectroelectrochemistry was performed under constant potential of $0.507 \mathrm{~V} v s$. $\mathrm{Fc}^{+} / \mathrm{Fc}$. Upon returning to the neutral species $\left(E=-0.113 \mathrm{~V} v s . \mathrm{Fc}^{+} / \mathrm{Fc}\right)$, some other absorption features emerge (green dotted line). The inset shows cyclic voltammogram of TTN in $0.1 \mathrm{M} \mathrm{TBAPF}_{6}$ in acetonitrile.

Our next objective was to investigate the absorption characteristics of $\mathrm{TTF}^{\bullet+}$, $\mathrm{TMT}_{-} \mathrm{TTF}^{\bullet+}$, and $\mathrm{TTN}^{\bullet+}$ in the gas phase. Action spectroscopy was performed at the electrostatic ion storage ring in Aarhus, ELISA. A detailed experimental description is found elsewhere. ${ }^{9}$ Briefly, ions were formed by electrospray ionization, accumulated in a 22-pole ion trap, accelerated as a bunch to 22-keV kinetic energy, mass-to-charge selected by a bending magnet and injected into the ring. After $35 \mathrm{~ms}$ in the ring, to allow for the decay of metastable ions, the ions were photoexcited with a pulsed tunable EKSPLA laser (Nd:YAG in combination with an OPO). The number of neutrals formed on one side of the ring was measured as a function of time. Dissociation was a result of one-photon absorption. Absorption cross-sections (relative, not absolute, numbers) are obtained as the number of neutrals formed after photoexcitation divided by the ion beam intensity and the number of photons in the laser pulse. The resulting gas-phase action spectra are shown in Figure 2.

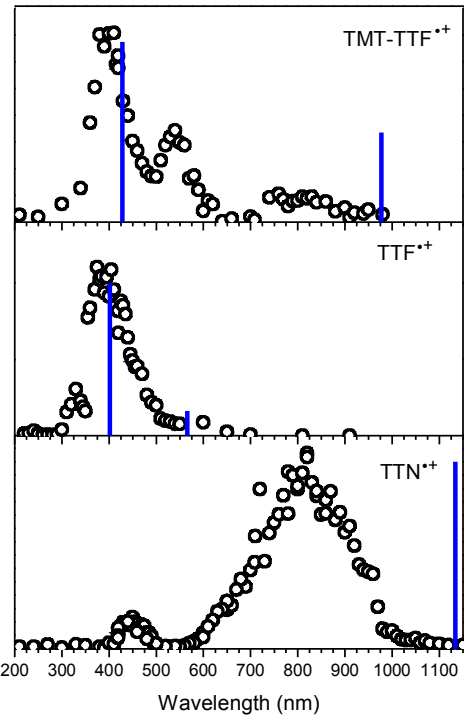

Figure 2. Gas phase action spectra and calculated values (vertical lines).

Theoretical results for the lowest-energy absorption of each radical cation species are presented in the figure as the solid blue vertical lines and a nice agreement is seen in the relative values though not in the absolute values. Time Dependent Density Functional Theory (TDDFT) in a time-propagating real-space formalism in the Local Spin Density Approximation (LSDA) with Perdew-Zunger Exchange-Correlation was used. ${ }^{10}$ The radius of the simulation box was $5.0 \AA$ and the grid spacing $0.19 \AA$, the total propagating time was $50 \mathrm{~h}-\mathrm{bar} / \mathrm{eV}$ equivalent to $33 \mathrm{fs}$. The structures were optimized on the B3LYP/6-311++G(d,p) level of theory. The solution phase, gas phase and theoretical results are summarized in Table 1.
Table 1. Absorption maxima (nm).

\begin{tabular}{cccc}
\hline Compound & Solution $(\mathrm{MeCN})$ & \multicolumn{2}{c}{ Gas phase } \\
& & Experiment & Theory \\
\hline TTF $^{\bullet+}$ & $580,714^{\text {a) }}$ & $395, \sim 600$ & 401,566 \\
TMT-TTF $^{\bullet+}$ & $843^{\text {b) }}$ & $398,540,820$ & 428,977 \\
TTN $^{\bullet+}$ & 900 & 450,815 & 1134 \\
FDTF $^{\bullet+}$ & -- & -- & 792 \\
\hline
\end{tabular}

a) In EtOH at low temperature. B) Ref. 7c.

Figure 3 shows the highest fully occupied orbital, SOMO-1, and the partial occupied orbital, SOMO, of the four radical cations. The transition from the SOMO- 1 to the SOMO is a $\pi-\pi^{*}$ transition for $\mathrm{TTF}^{\bullet+}, \mathrm{FDTF}^{\bullet+}$ and $\mathrm{TTN}^{\bullet+}$ where charge density is moved to the central C-C bridge. For TMT-TTF ${ }^{\bullet+}$ the transition is a $n-\pi *$ transition where charge is moved from the external Sulfurmethylthio to the $\mathrm{C}-\mathrm{C}$ bridge.

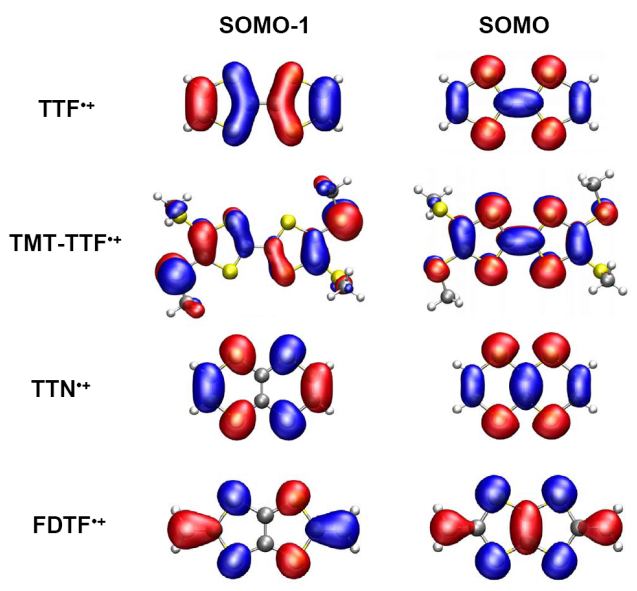

Figure 3. Kohn-Sham orbitals.

The gas-phase action spectrum of TMT-TTF ${ }^{\bullet+}$ displays three bands. The band at $398 \mathrm{~nm}$ is almost identical to the band in $\mathrm{TTF}^{\bullet+}$ which indicates a similar $\pi-\pi^{*}$ transition confined to the ring entity. The low-energy band at $815 \mathrm{~nm}$ is clear evidence that in solution the origin of this band is from an intramolecular transition and not the result of $\pi$-dimers, in agreement with the work by Khodorkovsky et al. (FOOTNOTE Any doubly charged dimers are unlikely to be present in the ion beam but they would in any case Coulomb explode into monocations after photoexcitation, which are not detected in the experiment.) The position at lower energy compared to the $\pi-\pi *$ transition (398 nm), and the low oscillator strength is in nice agreement with the $\mathrm{n}-\pi^{*}$ character of the transition. The character of the middle band is unknown. The lowenergy absorption (ca. $714 \mathrm{~nm}$ ) observed in solution at low temperature of $\mathrm{TTF}^{\bullet+}$ is absent in the gas phase spectrum, which supports the interpretation of this band as a $\pi$-dimer absorption.

Gas phase and solution phase experiments as well as theory all show that the lowest-energy absorption of the isomer $\mathrm{TTN}^{\bullet+}$ compared to $\mathrm{TTF}^{\bullet+}$ is significantly redshifted (by $215 \mathrm{~nm}$ in gas phase). This redshift is a measure of the effect of changing the central double bond from an exocyclic, fulvalene structure (TTF) to an endocyclic, fused ring structure (TTN). An expected solvatochromic redshift is found for the $\pi-\pi^{*}$ transitions in $\mathrm{TTF}^{\bullet+}$ and $\mathrm{TTN}^{\bullet+}$, whereas the shift of the $\mathrm{n}-\pi^{*}$ transition for TMT$\mathrm{TTF}^{\bullet+}$ is more complex. 
In conclusion this combined theoretical and experimental study provides the first gas phase absorption spectra, and hence intrinsic optical properties, of $\mathrm{TTF}^{\bullet+}$, $\mathrm{TMT}^{-\mathrm{TTF}^{\bullet+}}$, and $^{\mathrm{TTN}}{ }^{\bullet+}$ and the first solution phase absorption spectrum of $\mathrm{TTN}^{\bullet+}$. The results reveal unambiguously that the low-energy absorption of TMT-TTF and TNT racial cations in solution should not be assigned to $\pi$-dimers.

Acknowledgement. The Aarhus and Copenhagen groups gratefully acknowledge support from Lundbeckfonden. DV acknowledges funding provided by the European Research Council project n. 240624. D.V., L.A.E. and A.R. acknowledge eI3 ETSF project (Contract Number 211956), and CINECA CPU time granted through ISCRA. L.A.E. and A.R. acknowledge also the finantial support by MEC (FIS2007-65702-C02-01), ACIPromociona (ACI2009-1036), "Grupos Consolidados UPV/EHU del Gobierno Vasco" (IT-319-07) and the computational time granted by the Barcelona Supercomputing Center, "Red Española de Supercomputacion" and ARINA cluster.

\section{References}

(1) a) Bryce, M. R. Adv. Mater. 1999, 11, 11; b) Nielsen, M. B.; Lomholt, C.; Becher, J. Chem. Soc. Rev. 2000, 29, 153; c) Bryce, M. R. J. Mater. Chem. 2000, 10, 589; d) Segura, J. L.; Martín, N. Angew. Chem. Int. Ed. 2001, 40 ,
1372; d) Stoddart, J. F. Chem. Soc. Rev. 2009, 38, 1802; e) Canevet, D.; Sallé, M.; Zhang, G. X.; Zhang, D.; Zhu, D. Chem. Commun. 2009, 2245.

(2) Huchet, L.; Akoudad, S.; Levillain, E.; Roncali, J.; Emge, A.; Bäuerle, P.; J. Phys. Chem. B 1998, 102, 7776 .

(3) Torrance, J. B.; Scott, B. A.; Welber, B.; Kaufman, F. B.; Seiden, P. E. Phys. Rev. B 1979, 19, 730 .

(4) Khodokovsky, V.; Shapiro, L.; Krief, P.; Shames, A.; Mabon, G.; Gorgues, A.; Giffard, M. Chem. Commun. 2001, 2736.

(5) Lyskawa, J.; Sallé, M.; Balandier, J.-Y.; Le Derf, F.; Levillain, E.; Alain, M.; Viel, P.; Palacin, S. Chem. Commun. 2006, 2233.

(6) Aprahamian, I.; Olsen, J.-C., Trabolsi, A.; Stoddart, J. F. J. Am. Chem. Soc. 2008, 14, 3889 .

(7) a) Bryce, M. R.; Devonport, W.; Goldenberg, L. M.; Wang, C. Chem. Commun. 1998, 945; b) Christensen, C. A.; Goldenberg, L. M.; Bryce, M. R.; Becher, J. Chem. Commun. 2000, 331; c) Spanggaard, H.; Prehn, J.; Nielsen,

M. B.; Levillain, E.; Allain, M.; Becher, J. J. Am. Chem. Soc. 2000, 122, 9486 (8) Mizuno, M.; Cava, M. P.; Garito, A. F. J. Org. Chem. 1976, 41, 1485.

(9) a) Møller, S. P.; Nucl. Instrum. Meth.A 1997, 394, 281; b) Andersen, J. U; Hvelplund, P.; Brøndsted Nielsen, S.;Tomita, S.; Wahlgreen H.; Møller, S. P.; Pedersen, U. V.; Forster, J. S.; Jørgensen, T. J. D. Rev. Sci. Instrum. 2002, 73, 1284; c) Brøndsted Nielsen, S.; Andersen, J. U.; Hvelplund, P.; Liu, B.;

Tomita, S. J. Phys. B: At. Mol. Opt. 2004, 37, R25.

(10) Castro, A.; Appel, H.; Oliveira, M.; Rozzi, C. A.; Andrade, X.; Lorenzen, F.; Marques, M. A. L.; Gross, E. K. U.; Rubio, A. Phys. Stat. Sol. B 2006, 243 2465. 
